\title{
The ultrastructure of the Aedes aegypti heart
}

\author{
Ana Carolina M. Leódido ${ }^{a}$, Marcelo Ramalho-Ortigão ${ }^{\mathrm{b}}$, Gustavo F. Martins ${ }^{\mathrm{a}, *}$ \\ a Programa de Pós-graduação em Biologia Celular e Estrutural, Departamento de Biologia Geral, Universidade Federal de Viçosa (DBG/UFV), \\ Campus Universitário, Viçosa, Minas Gerais CEP 36570-000, Brazil \\ ${ }^{\mathrm{b}}$ Department of Entomology, Kansas State University (KSU), Manhattan, KS 66506, USA
}

\section{A R T I C L E I N F O}

\section{Article history:}

Received 26 January 2013

Received in revised form

13 September 2013

Accepted 18 September 2013

\section{Keywords:}

Aedes aegypti

Alary muscles

Cardiomyocytes

Heart

Mosquito

Pericardial cells

\begin{abstract}
A B S T R A C T
Comparative structural analyses of the heart and associated tissues in 4th instar larvae (L4), pupae and adults of Aedes aegypti were undertaken using a combination of microscopy techniques. The Ae. aegypti heart consists of cardiomyocytes arranged in a helical fashion, and it is physically associated with intersegmental groups of pericardial cells (PCs) and the alary muscles (AMs). Ramifications commonly present in AMs are more developed in adults than in the immature stages. Pericardial cells absorb and store extracellular components as shown by the uptake of carmine dye fed in larval diet. We also observed that carmine stained inclusions corresponding to electron-dense structures resembling lysosomes that were more abundant and prominent in pupae, suggestive of increase of waste accumulation during pupation. The results presented here expand on previously known aspects of the mosquito heart and describe for the first time comparative aspects of the morphology of the heart in different developmental stages.
\end{abstract}

๑ 2013 Elsevier Ltd. All rights reserved.

\section{Introduction}

Insects have an open circulatory system that allows communication between tissues and facilitates the transport of nutrients, waste metabolites, hemocytes, and humoral factors. These functions are accomplished via hemolymph circulation driven by the pumping action of a dorsal vessel. The dorsal vessel is divided into a contractile portion (heart) located in the abdomen, and a noncontractile portion (aorta) located in the thorax and head of the insect. Hemolymph reaches the heart lumen through segmentally arranged paired openings, or ostia, and is then pumped in the direction of the insect's head [reviewed by Snodgrass (1993) and Chapman et al. (2012)].

Pathogen invasion of the salivary glands is a prerequisite for the transmission of most vector-borne infectious diseases. These pathogens reach the salivary glands by disseminating through the hemolymph. Few studies have shown how pathogens are able to navigate the complex arthropod circulatory system that involves the insect heart and the pumping of hemolymph. Hillyer et al. (2007) demonstrated that Plasmodium berghei sporozoites rapidly flow with the hemolymph towards the anterior body portion of Anopheles gambiae, while some appear to be stationary in the ostia

\footnotetext{
* Corresponding author. Tel.: +55 313899 3492; fax: +55 3138992549.

E-mail addresses: anacarolinaleodido@hotmail.com (A.C.M. Leódido) mortigao@ksu.edu (M. Ramalho-Ortigão),gmartins@ufv.br (G.F. Martins).
}

that also coincide with the location of pericardial cells (PCs). Subsequently, Andereck et al. (2010) and Glenn et al. (2010) provided detailed descriptions of the heart in An. gambiae, and using fluorescent particles they were able to infer how pathogens navigate through this system. Later, King and Hillyer (2012) also demonstrated that pathogens are actually trapped by periostial hemocytes (cells that accumulate in the areas surrounding the heart's ostia) forming a phagocytic focus in regions of high hemolymph flow, which are also in the vicinity of the PCs. These results indicated that the circulatory system and immune system in An. gambiae are physiologically interrelated.

In contrast, not much is known about how viruses, particularly dengue, are transported through the hemolymph in adult Aedes aegypti and reach the salivary gland in this vector. In addition, only scarce details are available regarding the microanatomy of the adult heart of mosquitoes and even less is known about the morphology of the heart in immature stages (i.e. larvae and pupae).

Generally speaking two different cell types occur in close association with the insect heart, the PCs (also commonly refereed as nephrocytes) and the alary muscles (AMs). In addition to PCs and AMs, a third cell type, the periostial hemocytes were recently described as associated with the heart in An. gambiae (King and Hillyer, 2012), but not yet reported in other insects. Pericardial cells are thought to play a role in homeostasis through the filtration of the hemolymph (Sanger and McCann, 1968a; Brehblin and Hoffmann, 1980; Das et al., 2008a, 2008b). The AMs, which 


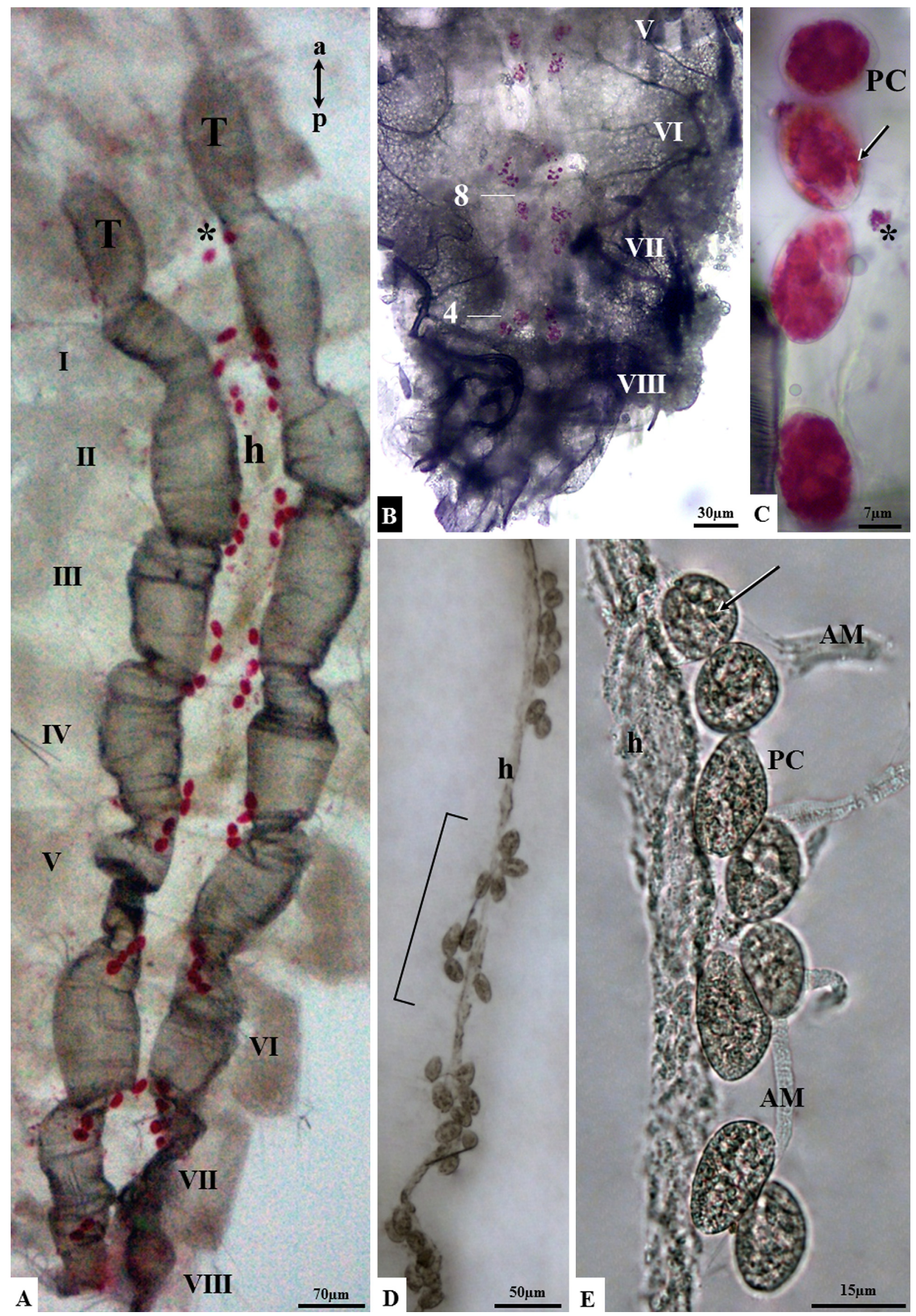


extend from the body wall to the heart and are physically attached to the surface of the heart via ramifications (myofibrils), form the suspensory apparatus likely associated with the pumping of hemolymph (Dulcis and Levine, 2003; Ejaz and Lange, 2008; Martins et al., 2011). In this study we focused our analyses on heart morphology in different stages of development of Ae. aegypti. We compared the structural organization of the heart in 4th instar larvae (L4), and in early pupae and young adult female mosquitoes. Our results expand on previous observations of the Ae. aegypti heart offering important insights into the morphology of the organ in larval and pupal stages of this mosquito.

\section{Materials and methods}

Ae. aegypti (strain PP - Campos, Campos dos Goytacazes, RJ, Brazil) were obtained from a colony maintained in the Departamento de Biologia Geral, Universidade Federal de Viçosa, MG, Brazil. Ae. aegypti larvae were maintained in dechlorinated tap water and fed with turtle food $\left(\right.$ Tetramin $\left.^{\circledR}\right)$ with a photoperiod of $12 \mathrm{~h} / 12 \mathrm{~h}$ at $25 \pm 3{ }^{\circ} \mathrm{C}$. Adult mosquitoes were kept at the same temperature and light-dark cycle with $55-65 \%$ relative humidity and fed on $20 \%$ sucrose solution ad libitum.

Hearts were dissected from L4, pupae ( $24 \mathrm{~h}$ after pupation) and adults (24 h after emergence). Prior to dissection, L4 and pupae were anesthetized in cold water, and adult females were placed at $4{ }^{\circ} \mathrm{C}$. All insects were kept on ice during dissections and each specimen was dissected separately under a stereomicroscope in $0.1 \mathrm{M}$ sodium phosphate buffer (PBS), $\mathrm{pH}$ 7.2.

\subsection{Whole mounts}

To facilitate visualization of PCs within mosquito whole mounts, powdered carmine was fed to L4 larvae $24 \mathrm{~h}$ prior to dissection. In addition, pupae and adults developed from carmine-fed larvae were also dissected. Ingestion of carmine stain was confirmed by the mosquito's midgut red color just after dissections. Three samples per developmental stage were used, the thoracic and abdominal cavities were opened laterally using a micro scissor (Petrovich Instrumentos Cirúrgicos, São Paulo, Brazil), and the visceral organs were removed; $4 \%$ paraformaldehyde in $0.1 \mathrm{M}$ in $\mathrm{PBS}, \mathrm{pH} 7.2$ was dripped directly onto each mosquito carcass.

For the "non-stained samples", after fixation, the heart and associated tissue were gently removed from the abdomen with the aid of forceps, transferred to glass slides, mounted in PBS and covered with coverslip. Samples were observed and photographed using an optical Olympus BX60 microscope coupled with digital Olympus Q-Color3 camera.

\subsection{Histology}

For the light microscopy analyses, at least 20 individuals from each developmental stage (L4 and pupae) were processed as follows. Each abdomen was separated from the thorax and cut laterally, and the visceral organs were removed. After dissection, samples were transferred to microcentrifuge tubes containing fixative solution for at least $2 \mathrm{~h}$. Fixed samples were rinsed in PBS, dehydrated in crescent series of ethanol (70\%-100\%), and embedded in JB4 Historesin (EMS) according to the manufacturer's instructions. Samples were sectioned $(3-4 \mu \mathrm{m})$ with glass knives in a microtome. Sections were stained with hematoxylin and eosin (HE), mounted in Eukitt (Fluka) mounting medium and photographed with an optical Zeiss Primo Star microscope coupled with a digital AxioCamERc5s camera.

\subsection{Morphometry}

Total areas of cytoplasm and nucleus of PCs were determined with Image ProPlus ${ }^{\mathrm{TM}}$ software. A total of 90 cell sections from 13 individuals (four L4, three pupae and five adults) were measured in the histological sections. Only sections in which the PCs appeared with the nucleus or nuclei were considered for measuring. Mean values of the two areas analyzed were compared using ANOVA and Tukey test. Results were deemed significant when $p<0.05$.

\subsection{Confocal laser scanning microscopy (CLSM)}

For CLSM, samples were fixed in $4 \%$ formaldehyde solution in PBS for a period of $30 \mathrm{~min}$ and washed three times in PBS, $5 \mathrm{~min}$ each. They were then incubated for $30 \mathrm{~min}$ in fluorescent Phalloidin-FITC (Sigma-Aldrich) diluted 1:100 in 0.1\% Triton X-100 and washed as before. Prior to analysis, samples were mounted with the anti-fading solution Mowiol (Fluka). After cytoskeleton stain, a number of samples were washed and incubated in Topro-3 Iodide (Invitrogen) 1:500 in PBS for $30 \mathrm{~min}$ for nuclei staining and washed again in PBS. Samples were protected from light during preparation. CLSM images were obtained from multiple confocal sections using a LCM510 laser confocal microscope (Zeiss).

\subsection{Scanning and transmission electron microscopy (SEM and TEM)}

For SEM and TEM analyzes, samples were transferred to a fixative solution containing $2.5 \%$ glutaraldehyde, $0.1 \mathrm{M}$ cacodylate buffer, pH 7.2 after dissection. Glutaraldehyde-fixed samples were post-fixed in $1 \%$ osmium tetroxide in $0.1 \mathrm{M}$ sodium cacodylate buffer pH 7.2 during $2 \mathrm{~h}$ in the dark.

For SEM, samples were dehydrated in a crescent series (30\%$100 \%$ ) of acetone, critical-point dried using $\mathrm{CO}_{2}$ and sputter coated with gold for observation under a LEO 1430VP (SEM). For TEM, after post-fixation, the isolated hearts were embedded in Spurr's resin (EMS, Hatfield, PA) according to manufacturer instructions. Ultrathin sections were stained with uranyl acetate and lead citrate, washed in distilled water, and analyzed under a Zeiss EM109 (TEM).

\section{Results}

\subsection{General morphology of the heart and associated cells}

The heart in Ae. aegypti L4, pupa and adult consists of a dorsal vessel that spans the abdomen and it is held on the abdominal wall by alary muscles (AMs). Pericardial cells (PCs) are seen within the AMs. In dissected whole mounts PCs and AMs are closely attached to the heart surface, and these two cell types (PCs and AMs) occur as intersegmental groups of cells in the abdomen (Fig. 1A, D, E).

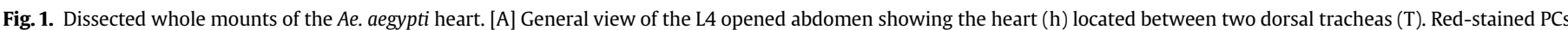

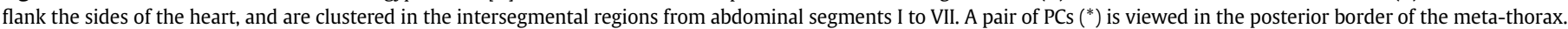

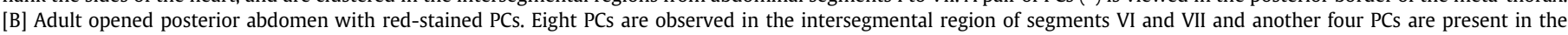

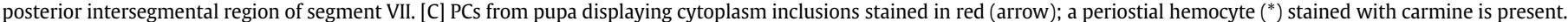

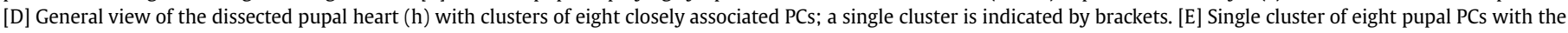
translucent AMs. The brown coloration of the PCs is due to the presence of cytoplasmic components (arrow) as indicated in C. a: anterior and p: posterior body regions. 


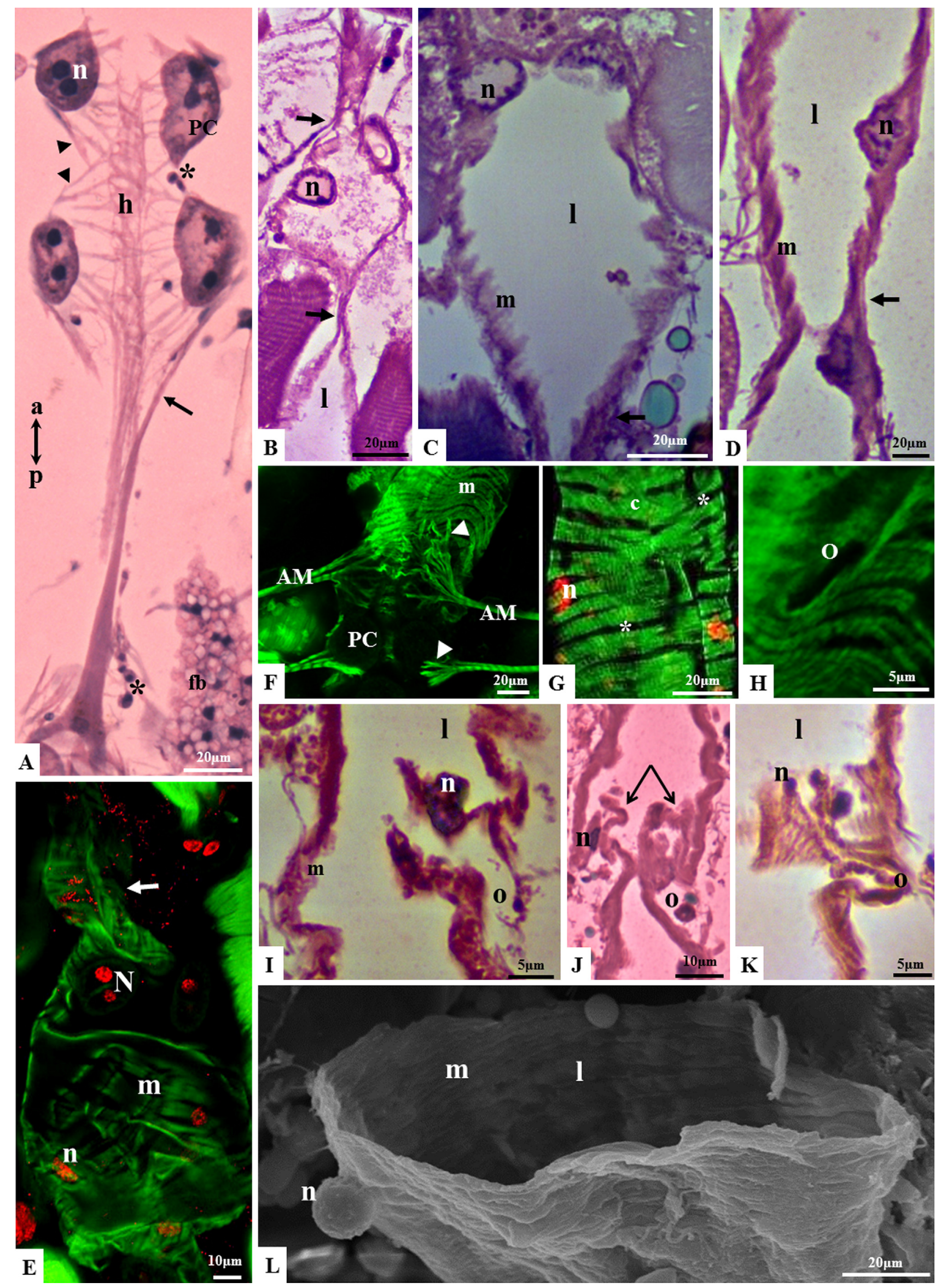


In histological sections, the PCs can be observed on both sides of the heart in Ae. aegypti, attached or closely associated to the heart wall. Every PC is trapped inside AM ramifications and each PC is flanked by one AM (Figs. 2A and 3A, D). In addition to these two cell types present in association with the mosquito heart, periostial hemocytes are commonly seen attached to AM ramifications, but mainly in adults. Some of these cells are also capable of internalizing the carmine dye (Fig. 1C). In contrast, AMs are translucent and are not visible in whole mounts in which the hearts are attached to the body wall (Fig. 1A, B); however, they can be seen at high magnification in dissected hearts (Fig. 1E).

\subsection{Structure of the heart musculature}

The muscular wall of the heart in Ae. aegypti (L4, pupae, and adults) consists of a single thin layer of transversely striated and acidophilic cardiomyocytes. In the three developmental stages, the heart thickness varies along its length seemingly due to its contractile or relaxed state (Fig. 2B-D). Also, in all three stages investigated, the cardiomyocytes can be seen circularly arranged on the inner and outer surfaces of the organ (Fig. 2E-G), especially on SEM preparations where the heart wall was broken (Fig. 2L). Protrusions corresponding to cellular cavities or cardiomyocytes nuclei were observed within the heart wall of L4 and pupae hearts (Figs. 2B, C). Using TEM, we observed that these cavities are lined with cardiomyocytes cytoplasm prolongations (Fig. 4A).

Cytoskeleton staining revealed that the Ae. aegypti heart is formed by strongly stained striated muscles during all three stages of development (Fig. 2E-G). Under the CLSM, interspaced individualized cardiomyocytes can be observed and cross striations of the sarcomeres are observed along their length (Fig. 2G). Spacing between fibers was likely caused by cell retraction and/or contraction during the fixation process.

SEM revealed that the surface of the heart in immature stages (L4 and pupa) differ from that of the adult. In the immature stages the heart has a ventral surface with a wrinkled appearance (Fig. 3A, $B$ ), while in adults the ventral surface of the heart has longitudinal grooves (Fig. 3C, D). Hearts of immature stages we examined are quite similar.

In the histological sections of the three stages investigated, ostia were not always detected. However, when detected, and regardless of the stage, they were present as invaginations of the heart wall in the intersegmental zones, co-occurring with the PCs. Our data suggest that a distinct subpopulation of cardiomyocytes differentiates into pairs of cells that form the ostia in Ae. aegypti L4, pupa and adult. Under the CLSM, although the ostia again were also not easily observed, they display a circular shape similar to a hole in the heart muscular wall in adults (Fig. $2 \mathrm{H}$ ). In the three developmental stages, the ostial cells form an opening in the dorsal vessel with their lips extending towards the cardiac lumen. Also, paired nuclei can be seen within the lip of these cells (Fig. 2I-K). The ostia appear as openings, with valves formed from projections of cardiac fibers into the heart (Fig. 2I, J). The ostial cells are also striated in a manner similar to the muscle cells of the heart wall (Figs. $2 \mathrm{~K}$ and $4 \mathrm{C}$ ).
TEM analysis shows that the Ae. aegypti myocardium has a variable thickness according to which region of the heart it is in, and also according to the developmental stages of the mosquito (Figs. $4 \mathrm{~A}-\mathrm{C}$ and $5 \mathrm{C}$ ), this could be due to the state of contraction at the time of fixation. Within cardiomyocytes one layer of myofilaments is seen and a single nucleus is always located at the cell periphery, protruding towards the inside or outside of the heart tube and with predominantly decondensed chromatin (Figs. 4A and $5 \mathrm{~A}, \mathrm{D})$.

Cardiomyocytes in L4, pupa and adult are bound by a thin basal lamina at the luminal and abluminal cell sides. Invaginations of the cell membrane appear as T-tubules (Fig. 4B). In addition, cardiomyocyte cytoplasm is filled with sarcomeres and these are separated by heavily stained discontinuous Z-lines forming the myofibril that, together with mitochondria, occupy almost the entire sarcoplasm (Figs. 4B, C and 5A-C). In cross-sectioned cardiomyocyte myofibrils, A- and I-bands appear as electron-dense and electron-lucent material, respectively (Fig. 5B). Large accumulations of predominantly rounded mitochondria, with a slightly electron-dense matrix, were observed in the periphery of the immature and adult cells (Figs. 4B and 5A).

\subsection{Pericardial cells (PCs)}

Fifty four PCs, usually arranged in 27 pairs, form two linear chains one on either side of the dorsal vessel in L4, pupa and adult Ae. aegypti. Out of the 54 PCs, 52 are displayed in L4, pupae and adults flanking the heart in the abdomen (Fig. 1A). In each of the abdominal intersegmental regions (from segments I through VII) four pairs of PCs, two pairs in one segment and two pairs in the subsequent segment, are present. Exclusively in the intersegmental region comprising segments VII and VIII only two pairs of PCs are present (in the posterior region of segment VII), as shown in Fig. 1A, $B$. An extra pair of PCs near the posterior border of the meta-thorax was only observed in L4 and pupa (Fig. 1A).

Adult mosquitoes were shown to retain carmine stain within the cytoplasm of their PCs, even after ecdysis and metamorphosis if fed as larvae (Pal, 1944). For carmine-fed individuals, all PCs in immature and adults are easily distinguished, having red-stained cytoplasm inclusions due to the dye uptake (Fig. 1A-C). In unstained individuals, components of the PC cytoplasm display a light-brown coloration (Fig. 1D, E).

In histological sections, L4, pupa and adult PCs appear as binucleated, mostly-round cells and are associated with the AMs in all abdominal segments (Figs. 2A and 3A, D, E). They display an oval shape with extensive light brown cytoplasmic inclusions containing carmine dye absorbed from the hemocoel (in case of carminefed mosquitoes). Over time the light brown coloration turns red (Fig. 1C, E).

With the HE stain, L4, pupa and adult PCs show spherical and very strong basophilic round-shaped cytoplasmic inclusions. The size and number of these structures change according to developmental stage, so that in the pupa these inclusions are larger and more abundant in comparison to the two other stages (Fig. 6A, B). These structures are also more abundant in adults than in larvae,

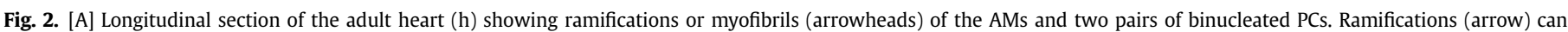

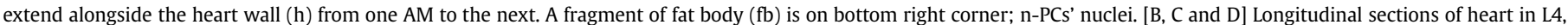

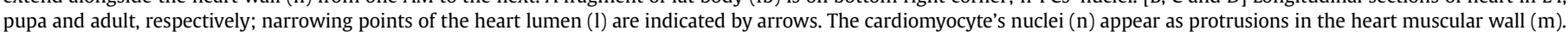

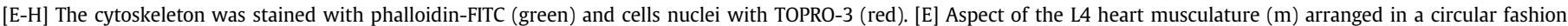

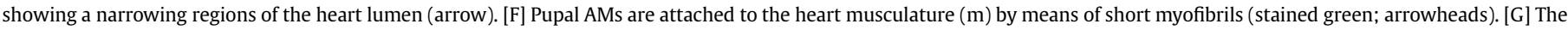

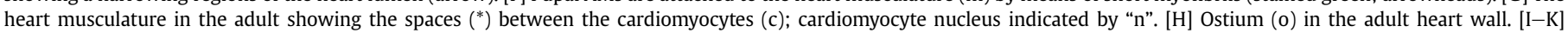

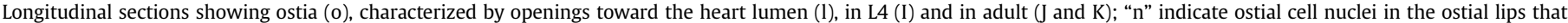

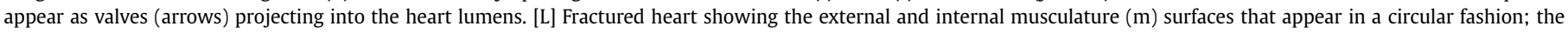
heart lumen (l) and the nucleus ( $\mathrm{n}$ ) of a cardiomyocyte are marked. a: anterior and p: posterior. 

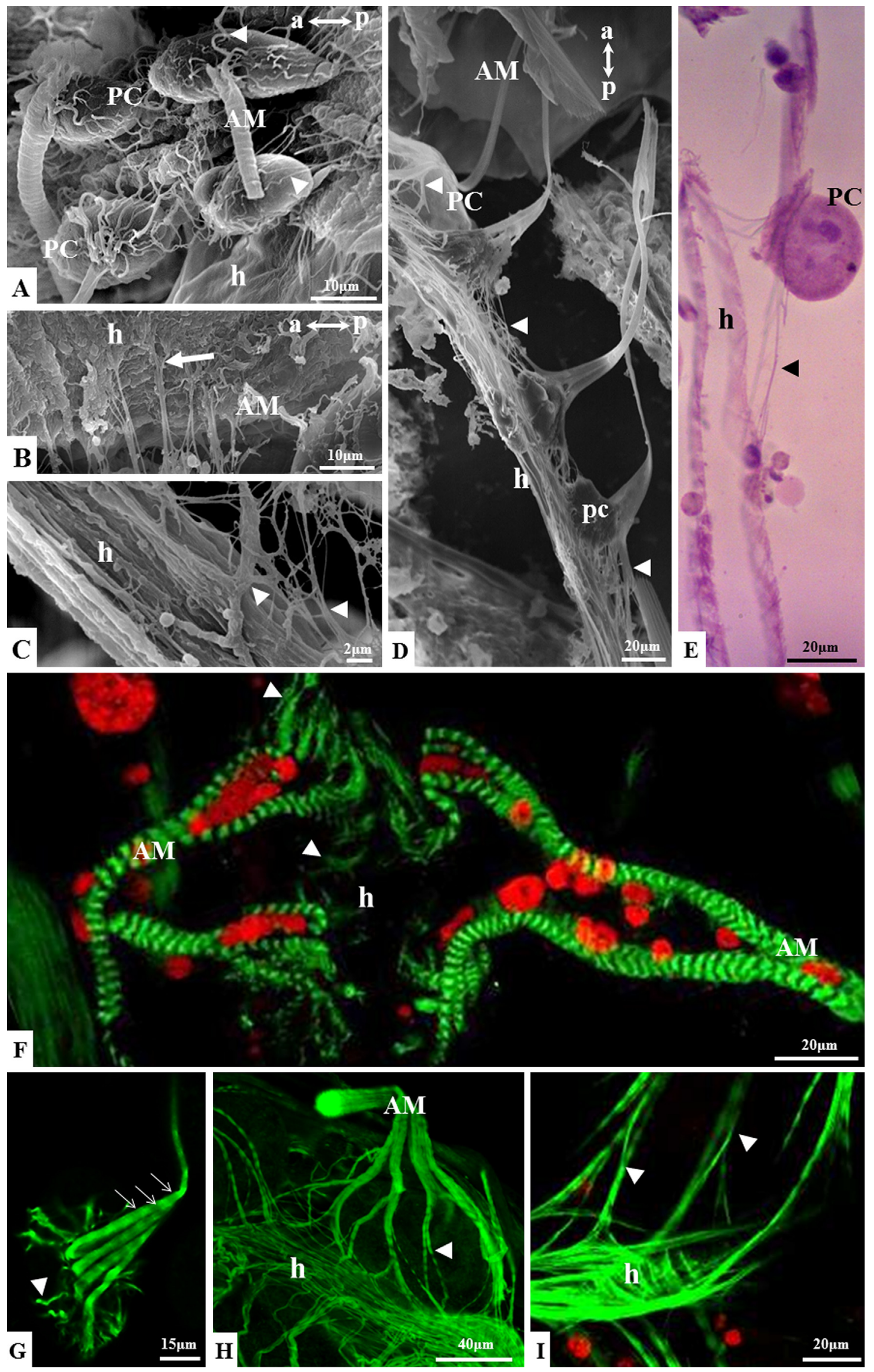
and under TEM they correspond to fairly large electron-dense structures resembling lysosomes (Fig. 6C, D).

In spite of PCs being in physical contact with the heart musculature and with $\mathrm{AM}$ ramifications (Figs. 1E, $2 \mathrm{~A}$ and $3 \mathrm{~A}, \mathrm{D}$ ), no specialized cell-junctions were observed between these structures in L4, pupa and adult hearts (Fig. 5B, D). Their nuclei have predominantly decondensed chromatin and prominent nucleoli (Fig. 6C, D). Also, PC ultrastructure is marked by the presence of electron-lucent vacuoles, and numerous coated vesicles and deep membrane invaginations into the cell cortex that form narrow channels filled with electron-dense granular material (Figs. 6C, D and 7D). Furthermore, the surface of these cells is covered by a basal lamina that does not accompany the membrane invaginations (Figs. 5D and 7D).

The L4, pupa and adult PCs' mitochondria are very electrondense and generally display elongated profiles (Figs. 6C and 7A). In the central portion of the cell cytoplasm prominent rough endoplasmic reticulum (RER), with enlarged lumen and Golgi elements are also commonly found (Fig. 7A, B).

Measurements of PCs' cytoplasm and nuclear cross-sectional areas indicated the former to be smaller in the L4 stage $(p<0.001)$, whereas they do not differ significantly from pupae and adults. In contrast, the nuclear area was greater in pupae $(p<0.001)$ in comparison to L4 and adults, and in these two stages no significant difference in size was observed. The measured areas for cytoplasm and nucleus for the PCs were as follows: for L4, the cytoplasm area measured $232.16 \pm 61.08 \mu \mathrm{m}^{2}$ and the nuclear area $18.91 \pm 4.44 \mu \mathrm{m}^{2}$; for pupae they were $345.90 \pm 91 \mu \mathrm{m}^{2}$ for cytoplasm and $23.08 \pm 3.46 \mu \mathrm{m}^{2}$ for nucleus; and for adults $309.69 \pm 55.12 \mu \mathrm{m}^{2}$ for cytoplasm and $19.54 \pm 3.34 \mu \mathrm{m}^{2}$ for nucleus.

\subsection{Alary muscles (AMs)}

Within Ae. aegypti abdominal segments I through VII, as indicated above, a single AM cell per PC seems to occur in immature and imago. Alary muscles are attached to the two sides of the heart and branch out in the form of ramifications or myofibrils forming a network that involves the PCs, and extend along the length of the heart wall in L4, pupa and adult hearts (Figs. 2A and 3A, D, E). The attachment and branching-out areas alternate with the PC clusters. Thus, the AMs attach to the heart on one side and to the body wall on the other.

In adult mosquitoes the AMs are also well developed and numerous compared to the immature stages (Figs. 2A and 3A, D, E). At the adult stage, AM ramifications extend along the heart's surface from one PC cluster to the next (Fig. 2A), and anastomose above the heart surface (Fig. 3C). Conversely, in the immature stages the AM ramifications are restricted to the vicinity of the PCs (Fig. $3 \mathrm{~A}, \mathrm{G}$ ) and they appear to stick to the basal lamina on the heart surface. Attachment points for AM ramifications are shown in Fig. 3B. Cross striation of the sarcomeres is observed along the AMs, including their ramifications in all studied mosquito stages (Figs. 3F-I and $7 \mathrm{C}$ ).

Alary muscles consist of multinucleated cells, and the nuclei are found throughout the whole cell but are concentrated in the transition between the cell body and the ramifications (Fig. 3F).

\section{Discussion}

Here, aspects of the ultrastructure of the heart and its associated cells in Ae. aegypti L4, pupae, and adults were compared by a combination of light microscopy, CLSM, SEM and TEM. The Ae. aegypti heart (or heart tube) runs along the dorsal side of the mosquito abdomen and is in close association with PCs and AMs. The heart consists of a single layer of cross-striated circular cardiomyocytes and this overall arrangement is maintained throughout the developmental stages investigated. Such circular cardiomyocyte organization appears to be conserved in insects, including Diptera (Jones, 1954; Cook and Meola, 1983; Angioy et al., 1999; Wasserthal, 1999; Glenn et al., 2010; Lehmacher et al., 2012), Lepidoptera (Sanger and McCann, 1968c), Blattaria (Edwards and Challice, 1960), Phasmatodea (Ejaz and Lange, 2008) and Heteroptera (Chiang et al., 1990).

Histology also showed that the heart of Ae. aegypti is divided into chambers or compartments that are separated by valves or incurrent ostia (Cook and Meola, 1983; Angioy et al., 1999). The ostia consist of heart openings where hemolymph inflow occurs (Angioy et al., 1999). In immature, ostia are simple openings in the heart wall, while in the adult they display a valve-like structure, appearing as an incurrent opening. Our results confirm and expand on previous observations that the architecture of the ostia, characterized by the presence of ostial lips, with the presence of sarcomeres, extending towards the cardiac lumen is identical in mosquito immature stages, adults, and are identical to those found in other dipterans (Curtis et al., 1999; Glenn et al., 2010; Lehmacher et al., 2012).

As shown in Drosophila melanogaster by Lehmacher et al. (2012), the mosquito cardiomyocytes also have large cavities lined with cytoplasmic-free membrane within them. In D. melanogaster such cavities are characteristic of the valve cells of immature and imago stages, and in Ae. aegypti they are found only in L4 and pupae. In spite of the fact that no roles have been formally assigned to these cavities, we hypothesize that function as valves. In support of this idea, in Diptera, several structures that project from the heart wall into the lumen of the dorsal vessel were suggested to work as heart valves (reviewed by Pass et al., 2006).

Not surprisingly, Ae. aegypti cardiomyocytes display thin and thick filaments with sarcomeres consisting of A- and I-bands and discontinuous Z-discs, as described for other insects (Sanger and McCann, 1968c; Sanger, 1979; Lehmacher et al., 2012). In addition, L4, pupa, and adult mosquito cardiomyocytes have a cytoplasmic membrane with T-tubules, as shown in D. melanogaster (Medioni et al., 2009). This membrane is thought to be the site where an electrical impulse induces the release of calcium ions by the sarcoplasmic reticulum allowing the nerve stimulus to reach the center of the muscle fibers, resulting in rapid and uniform contractions (Sanger, 1979; Curtis et al., 1999).

The number of abdominal pericardial cells (PCs) and the ratio of nuclei/cytoplasm were reported to vary among insect species, including mosquitoes and other dipterans (Pal, 1944; Jones, 1954; Denholm and Skaer, 2009). In mosquitoes, PCs are mostly binucleated and sometimes tri-nucleated (Pal, 1944), while in D. melanogaster they are mono-nucleated cells (Mills and King, 1965; Curtis et al., 1999). Additionally, according to our results

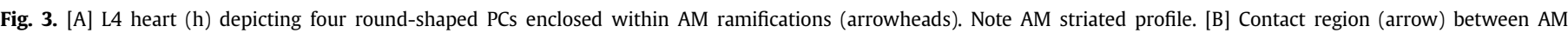

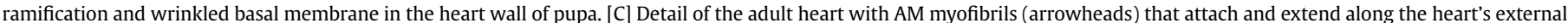

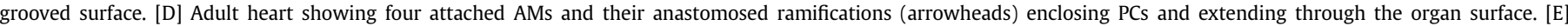

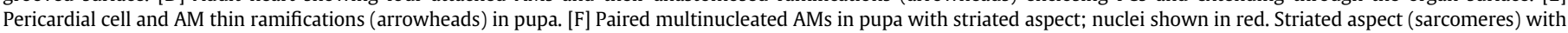

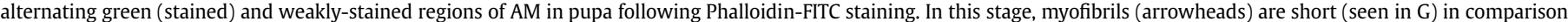

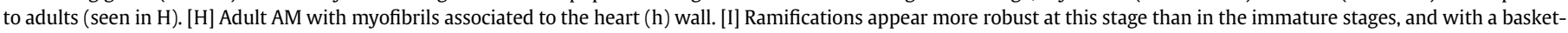
like appearance. a: anterior and p: posterior. (For color information please refer to the online version of the journal.) 

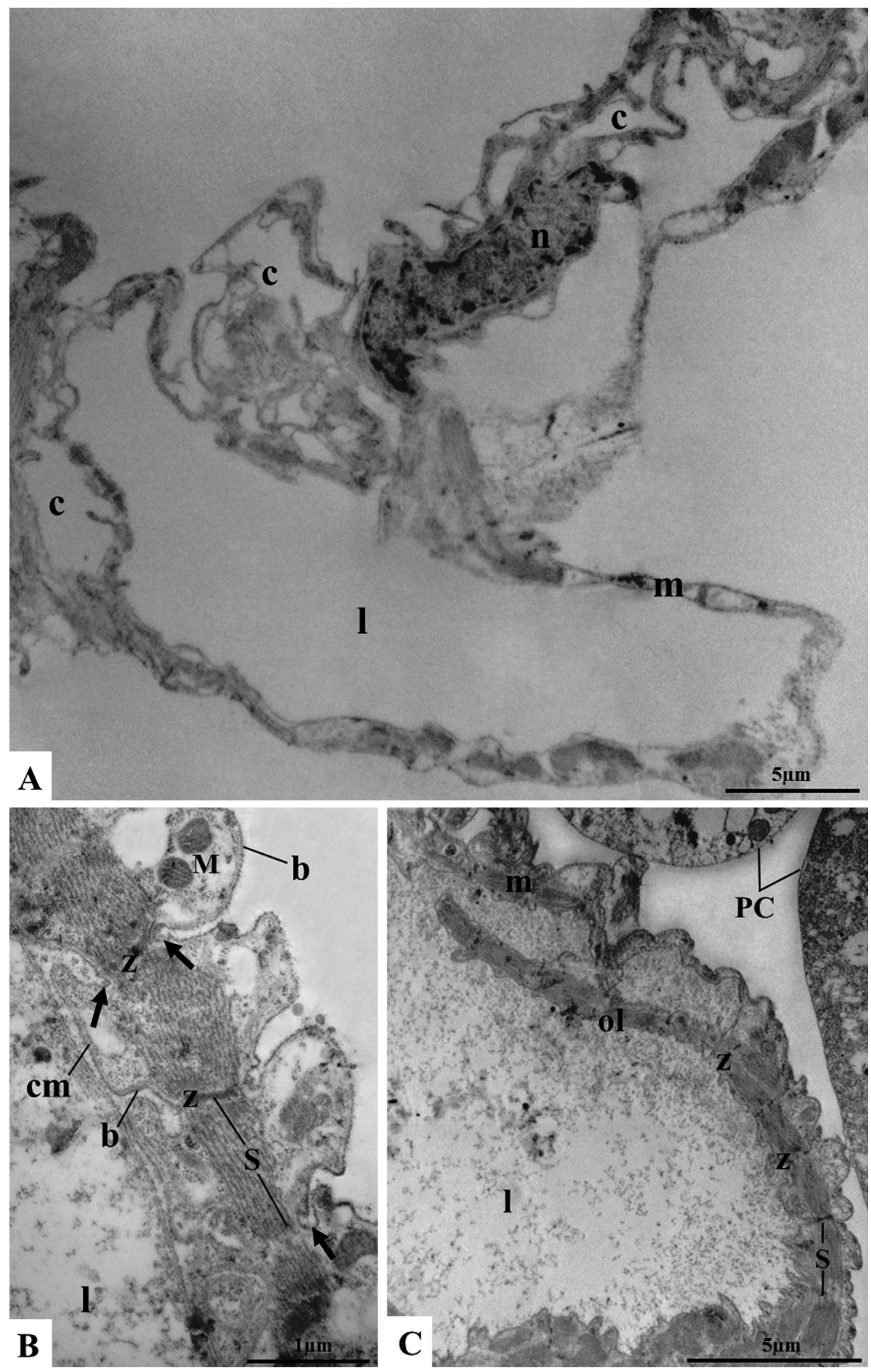

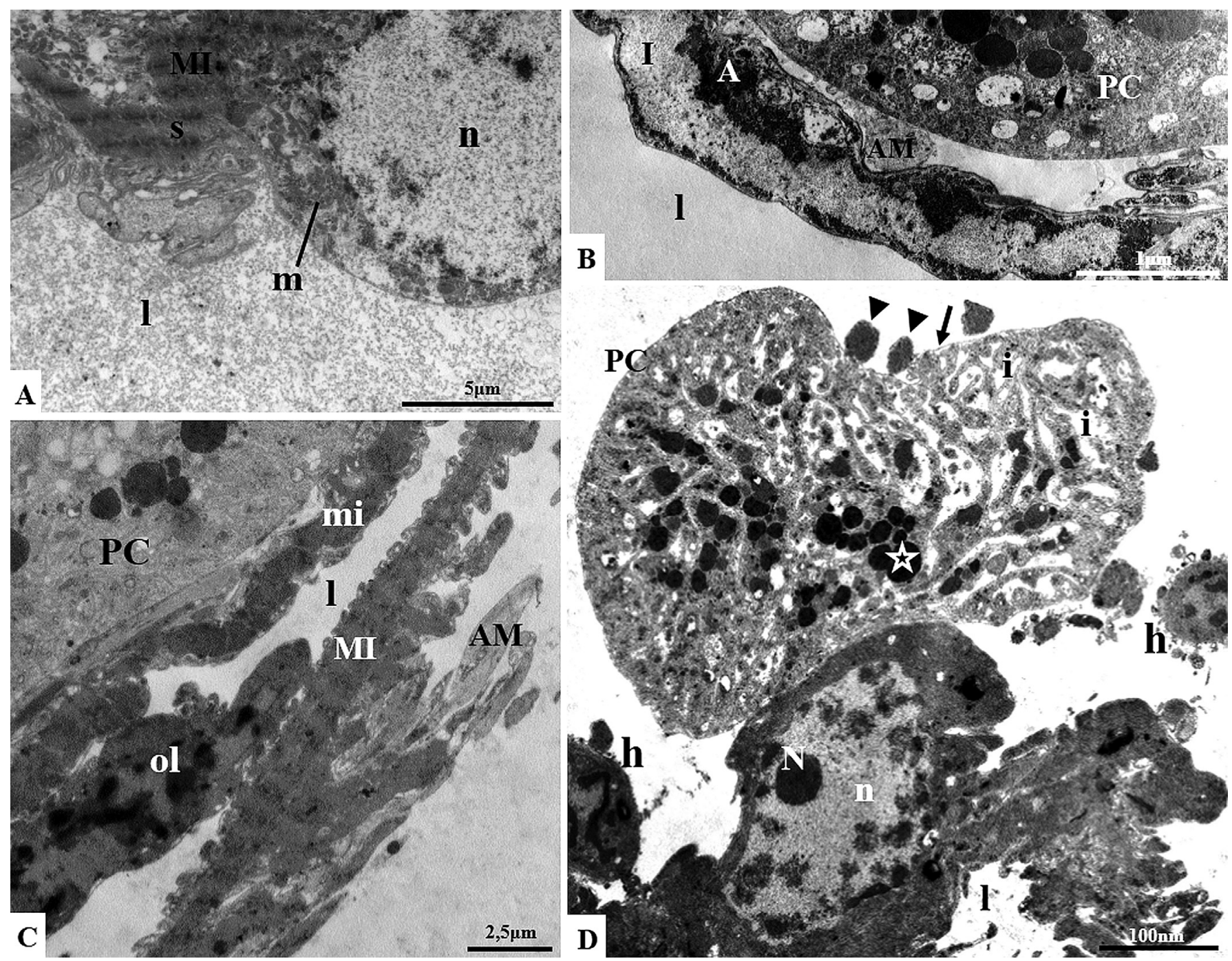

Fig. 5. TEM of the Ae. aegypti heart. [A] Pupal heart depicting a portion of a cardiomyocyte with mitochondria (m). [B] Pericardial cell cortex and the heart muscle with crosssectioned A- and I-bands in pupa are shown. Note the AM ramification located between these two structures. [C] Heart musculature depicting transversally (mi) and longitudinally cut (MI) myofibrils, with an ostial lip (ol); [D] PC cortex with cell membrane invaginations (i) and lysosome-like structures (star). Pericardial cell is enclosed by a basal lamina (arrow) and is in close contact with the protruded cardiomyocytes nucleus (n); Ramifications of the AM (arrowheads), the heart lumen (l), periostial hemocyte (h) and the nucleolus (N) are shown.

the number of abdominal PCs (i.e., 52) and the nuclei/PC ratio do not change in Ae. aegypti from larvae to adults.

Feeding of carmine dye to Ae. aegypti L4 in order to stain the PCs in all three developmental stages (L4, pupa, and adult) confirmed that these cells are capable of regulating hemolymph composition by filtering waste, as shown for several insects (Wigglesworth, 1943; Mills and King, 1965; Sanger and McCann, 1968a; Fife et al., 1987; Das et al., 2008a, 2008b; Denholm and Skaer, 2009; Hernández-Martínez et al., 2013). The scavenging capacity of PCs are thought to be important for the uptake of toxic macromolecules and colloids, thus functioning as classical nephrocytes sequestering endogenous and exogenous macromolecules from hemolymph (Das et al., 2008a, 2008c).

The PC plasma membrane produces a network of channels to facilitate absorption of extracellular material (Denholm and Skaer,
2009; Weavers et al., 2009). This complex network is present in Ae. aegypti, consistent with the proposed role for PCs in absorption of extracellular material in insect species (Bowers, 1964; Lehmacher et al., 2012; Hernández-Martínez et al., 2013). The Ae. aegypti PC cortex displays a high concentration of coated vesicles, likely the result of encapsulation of extracellular materials by the cytoplasmic membrane invaginations and internalization, as shown in D. melanogaster (Mills and King, 1965; Harrat et al., 2006). After the endocytic vesicles are formed they combine with enzymes produced in the RER giving rise to the lysosome-like electron-dense structure (Mills and King, 1965; Fife et al., 1987).

Ae. aegypti PCs are larger in pupae in comparison to adults and L4, and larger in adults than in L4. During metamorphosis major morpho-physiological and behavioral changes occur, thus storing substances absorbed from the hemolymph during the pupal stage

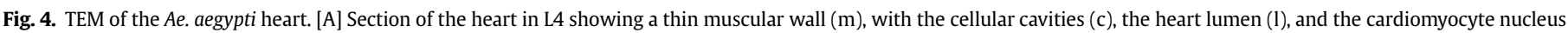

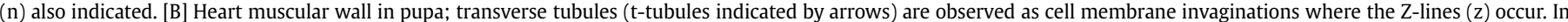

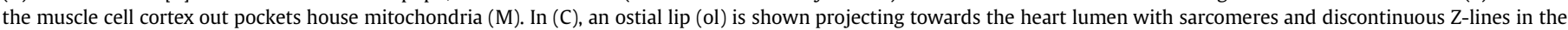
adult. 

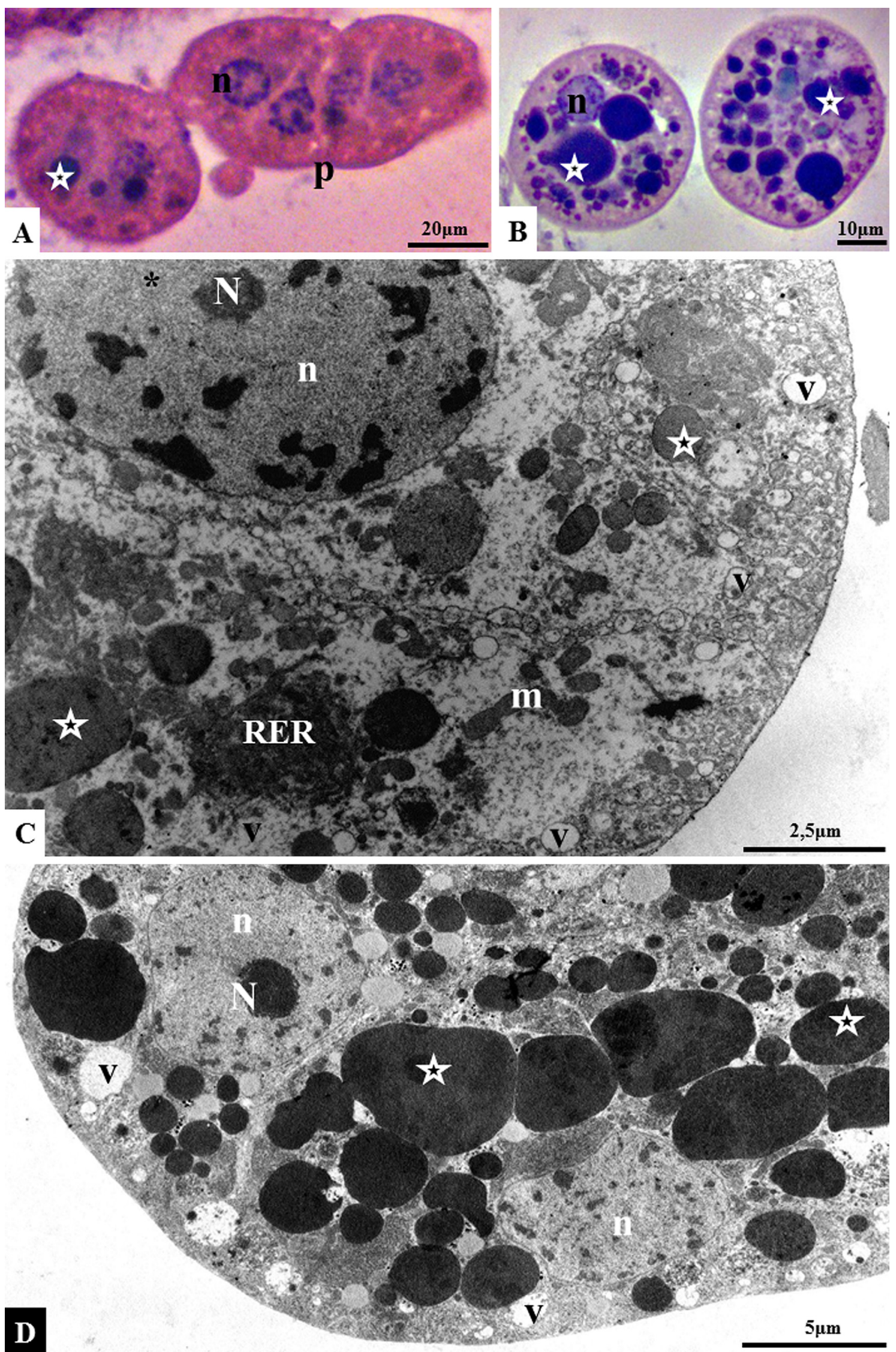

Fig. 6. Histology and TEM analyses of PCs. [A and B] Histological sections of Ae. aegypti PCs stained with hematoxylin and eosin; L4 (A) and pupa (B) PCs with highly basophilic cytoplasm components (stars), which are more abundant in pupa (B) and adult (not shown). [C and D] TEM micrographs of the Ae. aegypti PCs. [C] Pericardial cell of larva with 

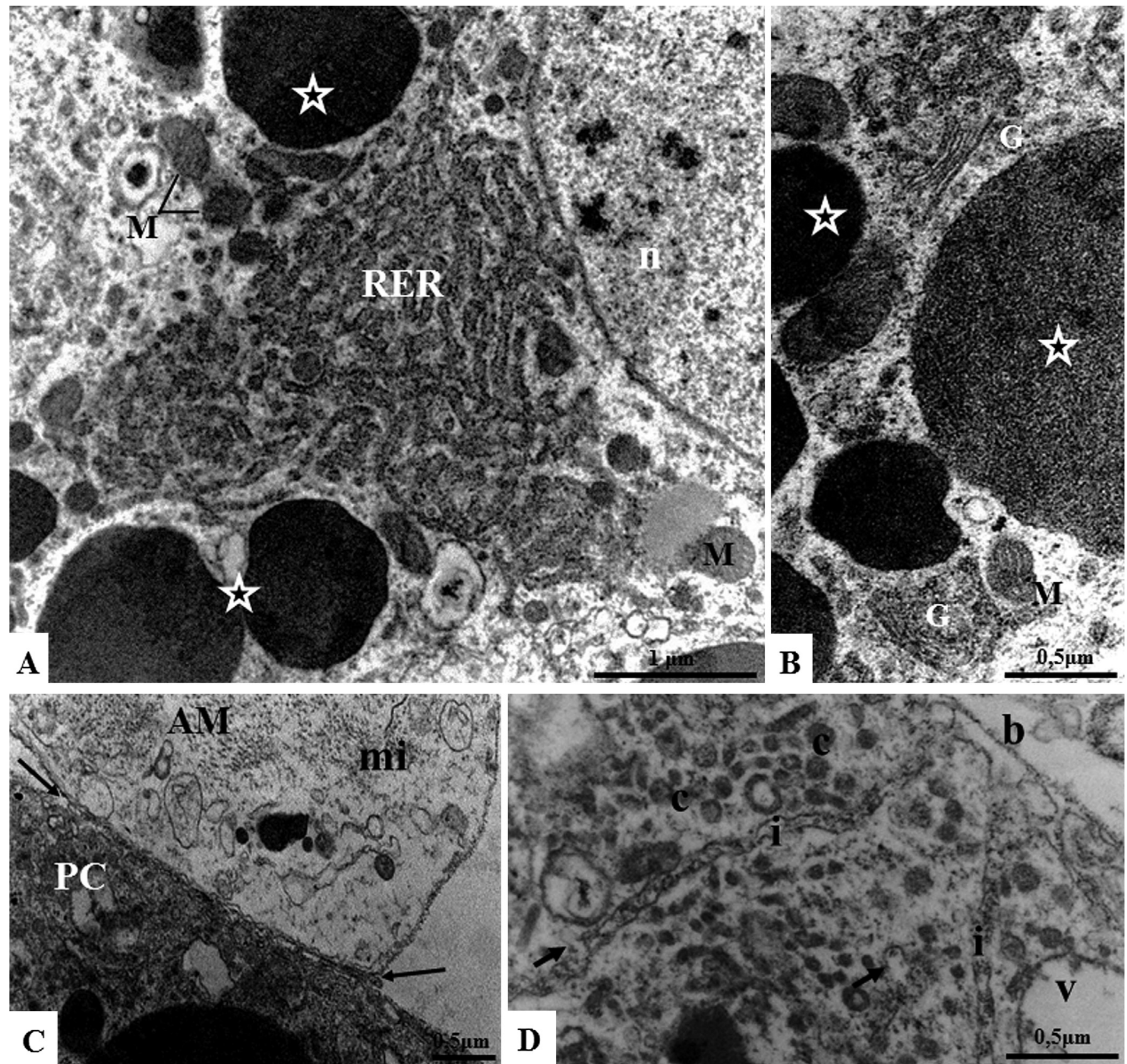

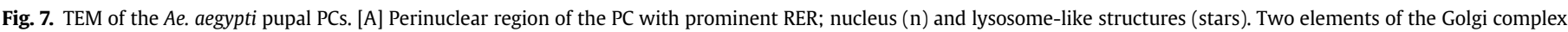

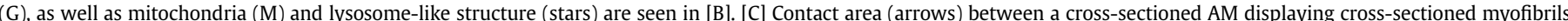

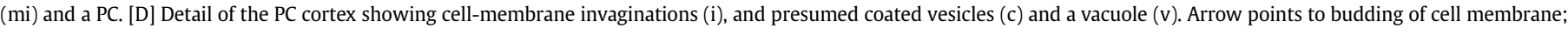
b - basal lamina.

may explain why the PCs in Ae. aegypti pupae have highly basophilic and electron-dense cytoplasmic inclusions that are larger and more abundant than in the other stages. It has been shown that lysosomal content in PCs turn electron-dense once the endocytosed material is digested (Mills and King, 1965; Harrat et al., 2006). Therefore, the increase in cytoplasmic inclusions in PCs during post-embryonic development of Ae. aegypti may consist of stored materials to be inactivated (Owa et al., 2010). However, it is not yet known whether endocytic activity followed by intracellular digestion leads to accumulation of stored lysosomal contents within PCs or how precisely these cells are involved with maintaining homeostasis.

In Ae. aegypti the heart is connected to the epidermis by a set of segmentally arranged alary muscles (AMs) that are inserted into the body wall at the intersegmental boundary and serve as a kind of diaphragm [Bodmer (1995) and references therein]. The number of

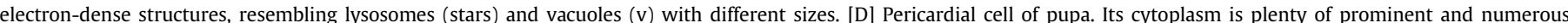

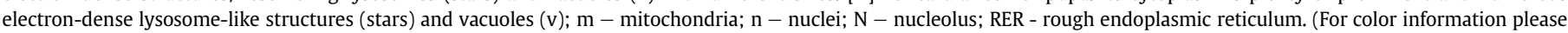
refer to the online version of the journal.) 
AM does not change during development in Ae. aegypti and under the CLSM these cells show a pattern of transverse striations on the cell body that is similar to observations made in An. gambiae (Andereck et al., 2010; Glenn et al., 2010) and in D. melanogaster (Lehmacher et al., 2012). Whereas in D. melanogaster the AMs are connected to the heart by a connective tissue that is part of the AM extracellular matrix (Curtis et al., 1999; Lehmacher et al., 2012), ramifications in Ae. aegypti AMs are cellular structures with striations corresponding to myofibrils (AM ramifications have thin and thick filaments under the TEM) and delimited by the cell membrane. These ramifications become more abundant and developed in adults, spreading alongside the heart's surface and reaching the next intersegmental region, suggestive of the robustness of the adult heart in comparison with immature stages. Conversely, in immature stages AM ramifications only extend slightly beyond the vicinity of nearby PCs. The development of AM ramifications in Ae. aegypti may be related to hemolymph pumping (Sanger and McCann, 1968b; Chiang et al., 1990). Accordingly, since the AMs have been hypothesized to be involved in the relaxation of the heart to promote entry of the hemolymph into the vessel, it is likely that the activity of these fibers is more intense in the winged adults. Moreover, adult mosquitoes do not display wriggling movements (flexing of the body in the water) used by immature (Strickman, 1989) to assist in hemolymph pumping. Wriggling may be a way for immature to compensate for a less robust heart than in adults.

Several factors may contribute to the inability of effectively controlling mosquito populations and preventing transmission of diseases such as dengue and malaria. The slow progress in developing effective therapies and vaccines combined with the ever growing issue of mosquito resistance to insecticides have contributed to the high prevalence of these and other mosquito-borne diseases. In this regard, clear understanding of underlying aspects of the mosquito biology will likely provide new tools to control these vectors. Thus, identifying what does happen during development to such vital organs such as the dorsal vessel (including the heart) and understanding cell function relationships of heartassociated cells can prove invaluable for mosquito control applications. In addition, the insect heart also has been suggested as a model for understanding basic physiological and morphological aspects of the vertebrate heart (Bodmer, 1995; Lehmacher et al., 2012; Na et al., 2013).

\section{Acknowledgments}

We would like to thank Dr. Julián Hillyer for comments of a previous version of this manuscript, to CAPES for financial support, and to the Center for Microscopy and Microanalysis (UFV) for technical support.

\section{References}

Andereck, J.W., King, J.G., Hillyer, J.F., 2010. Contraction of the ventral abdomen potentiates extracardiac retrograde hemolymph propulsion in the mosquito hemocoel. PLoS One 5, e12943.

Angioy, A.M., Boassa, D., Dulcis, D., 1999. Functional morphology of the dorsal vessel in the adult fly Protophormia terraenovae (Diptera, Calliphoridae). J. Morphol. 240, 15-31.

Bodmer, R., 1995. Heart development in Drosophila and its relationship to vertebrates. Trends Cardiovasc. Med. 5, 21-28.

Bowers, B., 1964. Coated vesicles in the pericardial cells of the aphid (Myzus persicae Sulz). Protoplasma 59, 351-367.

Brehblin, M., Hoffmann, J., 1980. Phagocytosis of inert particles in Locusta migratoria and Galleria mellonella: study of ultrastructure and clearance. J. Insect Physiol. 26, 103-111.

Chapman, R.F., Simpson, S.J., Douglas, A.E., 2012. The Insects: Structure and Function, fifth ed. Cambridge University Press, Cambridge.

Chiang, R., Chiang, J., Davey, K., 1990. Morphology of dorsal vessel in the abdomen of the blood-feeding insect Rhodnius prolixus. J. Morphol. 204, 9-23.
Cook, B.J., Meola, S., 1983. Heart structure and beat in the stable fly, Stomoxys calcitrans. Physiol. Entomol. 8, 139-149.

Curtis, N.J., Ringo, J.M., Dowse, H.B., 1999. Morphology of the pupal heart, adult heart and associate tissues in the fruit fly, Drosophila melanogaster. J. Morphol. 240, 225-235.

Das, D., Aradhya, R., Ashoka, D., Inamdar, M., 2008a. Post-embryonic pericardial cells of Drosophila are required for overcoming toxic stress but not for cardiac function or adult development. Cell Tissue Res. 331, 565-570.

Das, D., Aradhya, R., Ashoka, D., Inamdar, M., 2008b. Macromolecular uptake in Drosophila pericardial cells requires rudhira function. Exp. Cell Res. 314, 18041810.

Das, D., Ashoka, D., Aradhya, R., Inamdar, M., 2008c. Gene expression analysis in post-embryonic pericardial cells of Drosophila. Gene Expr. Patterns 8, 199-205.

Denholm, B., Skaer, H., 2009. Bringing together components of the fly renal system. Curr. Opin. Genet. Dev. 19, 526-532.

Dulcis, D., Levine, B., 2003. Innervation of the heart of the adult fruit fly Drosophila melanogaster. J. Comp. Neurol. 465, 560-578.

Edwards, G.A., Challice, C.E., 1960. The ultrastructure of the heart of the cockroach Blattella germanica. Ann. Entomol. Soc. Am. 5, 369-383.

Ejaz, A., Lange, A.B., 2008. Peptidergic control of the heart of the stick insect, Baculum extradentatum. Peptides 29, 214-225.

Fife, H.G., Palli, S.R., Locke, M., 1987. A function for pericardial cells in an insect Insect Biochem. 17, 829-840.

Glenn, J.D., King, J.G., Hillyer, J.F., 2010. Structural mechanics of the mosquito heart and its function in bidirectional hemolymph transport. J. Exp. Biol. 213, $541-$ 550.

Harrat, A., Schoeller-Raccaud, J., Ben-Hamouda, M.H., 2006. Evolution of the pericardial cells during embryogenesis without diapause in Locusta migratoria $L$. (Orthoptera-Acrididae). Tunis. J. Plant Prot. 1, 43-54.

Hernández-Martínez, S., Lanz-Mendoza, H., Martínez-Barnetche, J., Rodríguez, M.H., 2013. Antimicrobial properties of Anopheles albimanus pericardial cells. Cell Tissue Res. 351, 127-137.

Hillyer, J.F., Barreau, C., Vernick, K.D., 2007. Efficiency of salivary gland invasion by malaria sporozoites is controlled by rapid sporozoite destruction in the mosquito haemocoel. Int. J. Parasitol. 37, 673-681.

Jones, J.C., 1954. The heart and associated tissues of Anopheles quadrimaculatus Say (Diptera: Culicidae). J. Morphol. 94, 71-123.

King, J.G., Hillyer, J.F., 2012. Infection-induced interaction between the mosquito circulatory and immune systems. PLoS Pathog. 8, e1003058.

Lehmacher, C., Abeln, B., Paululat, A., 2012. The ultrastructure of Drosophila heart cells. Arthropod Struct. Dev, 41, 459-474.

Martins, G.F., Ramalho-Ortigão, J.M., Pimenta, P.F.P., 2011. Morphological features of the heart of six mosquito species by scanning electron microscopy. Int. J. Trop. Insect Sci. 31, 98-102.

Medioni, C., Sénatore, S., Salmand, P.A., Lalevée, N., Perrin, L., Sémériva, M., 2009 The fabulous destiny of the Drosophila heart. Curr. Opin. Genet. Dev. 19, 518525 .

Mills, R.P., King, R.C., 1965. The pericardial cells of Drosophila melanogaster. Q. J. Microsc. Sci. 106, 261e268.

Na, J., Musselman, L.P., Pendse, J., Baranski, T.J., Bodmer, R., Ocorr, K., Cagan, R., 2013. A Drosophila model of high sugar diet-induced cardiomyopathy. PloS Genet. 9, e1003175.

Owa, C., Aoki, F., Nagata, M., 2010. Protein degradation in silkworm peritrachea athrocytes and its physiological role in metamorphosis. J. Insect Physiol. 56, $1108-1117$.

Pal, R., 1944. Nephrocytes in some culicidae-diptera. Indian J. Entomol. 6, 143-148.

Pass, G., Gereben-Krenn, B.-A., Merl, M., Plant, J., Szucsich, N.U., Tögel, M., 2006. Phylogenetic relationships of the orders of Hexapoda: contributions from the circulatory organs for a morphological data matrix. Arthropod Syst. Phylogeny $64,165 \mathrm{e} 203$.

Sanger, J.W., McCann, F.V., 1968a. Fine structure of the pericardial cells of the moth, Hyalophora cecropia, and their role in protein uptake. J. Insect Physiol. 14, 18391845

Sanger, J.W., McCann, F.V., 1968b. Ultrastructure of moth alary muscles and their attachment to the heart wall. J. Insect Physiol. 14, 1539-1544.

Sanger, J.W., McCann, F.V., 1968c. Ultrastructure of the myocardium of the moth, Hyalophora cecropia. J. Insect Physiol. 14, 1105-1111.

Sanger, J.W., 1979. Cardiac fine structure in selected arthropods and molluscs. Am. Zool. 19, 9-27.

Snodgrass, R.E., 1993. Principles of Insect Morphology, second ed. Cornell University Press, New York.

Strickman, D., 1989. Biosystematics of larval movement of central American mosquitoes and its use for field identification. J. Am. Mosq. Control Assoc. 5, 208-218.

Wasserthal, L.T., 1999. Functional morphology of the heart and of a new cephalic pulsatile organ in the blowfly Calliphora vicina (Diptera: Calliphoridae) and their roles in hemolymph transport and tracheal ventilation. Int. J. Insect Morphol. Embryol. 28, 111-129.

Weavers, H., Prieto-Sánchez, S., Grawe, F., Garcia-López, A., Artero, R., WilschBraeuninger, M., Ruiz-Gómez, M., Skaer, H., Denholm, B., 2009. The insect nephrocyte is a podocyte-like cell with a filtration slit diaphragm. Nature 457 , $322-326$.

Wigglesworth, V.B., 1943. The fate of haemoglobin in Rhodnius prolixus (Hemiptera) and other blood-sucking arthropods. Proc. R. Soc. Lond. B Biol. Sci. 131, 313-339. 